\title{
Attribute Grammar Applied to Human Activities Recognition in Intelligent Environments
}

\author{
Leandro O. Freitas ${ }^{1}$, Pedro Rangel Henriques ${ }^{1}$, Paulo Novais ${ }^{1}$ \\ ${ }^{1}$ ALGORITMI Center, University of Minho, Braga, Portugal \\ leanfrts@gmail.com,\{prh,pjon\}@di.uminho.pt
}

\begin{abstract}
Keywords: Context-aware systems, human activities recognition, attribute grammar.
Abstract: Researches about context awareness have been growing in the past decades. The development of services that considers the context of users are getting popular and are gaining more functionalities, making them smarter. One of the most common features is the monitoring of activities through a diversity of sensors. Yet, this is still superficial monitoring where the devices lack information sharing. Intelligent environments aim the exchanging of information with the purpose of creating models that represent real-world situations. This paper describes the use of an attribute grammar in order to create a formal specification of situations in such domains. The problem of representation of human activities is tackled through a case study to demonstrate how attribute grammar can help the improvement of this process.
\end{abstract}

\section{Introduction}

Identification of patterns behaviour of users is considered an obstacle for the consolidation of intelligent environments. Systems for these domains should be aware of the users' steps along the day in order to identify what and how they perform their daily actions. Monitoring activities allow the system to improve its capability of assisting users by adjusting the environment according to their preferences or even helping them to solve problems. These systems have multiple agents capable of processing context data and sharing it with each other, aiming to increase the level of its intelligence. Context can be defined as anything that can influence the behaviour of users (Freitas et al., 2018).

One of the main problems when developing context-aware systems refers to the detection of environmental data, once this will be used as a premise for processing and understanding what happens in the domain. Thus, incomplete or lack of enough context data may compromise this process leading to uncertain situations, and consequently, the decision making by the system. Solutions for the real world should take into account different levels of uncertainty (White et al., 2018). The representation of it improves assertions about the domain's knowledge. This tends to minimize problems related to the design of the model and, consequently, the negative impact of misinterpretation of contexts (Anderson et al.,
2015).

Modelling human activities is not an easy task. It is necessary to develop means that allows the manipulation of context data and use it to create models that represent what happens in the environment. Besides that, it is necessary to validate this through a formal specification. Attribute Grammar (AG) (Knuth, 1968) is a field of investigation that can be applied for the validation of human activities recognition in intelligent environments due to its formalism.

Considering this, the main goal of this work is to describe how AGs can be used to tackle this problem. It is presented how they can improve the correctness of context data analysis. For this, the paper is structured as follows: section 2, describes concepts of AGs and how they can be applied to intelligent environments, once not much it is known about their relation. Section 3 presents an example of AG for human activities recognition aiming to demonstrate its usefulness. Related work is described in section 4 . The benefits of AGs applied to context-aware systems and final considerations of the paper are discussed in 5 .

\section{Attribute Grammars applied to Intelligent Environmnets}

Attribute Grammar (AG) is a wide field of investigation with several relevant contributions regarding 
the validation of programming languages. It introduces semantic rules into Context-Free Grammars, inserting meaning to the analysis of the grammar (Knuth, 1968). Still, according to (Knuth, 1968), the semantics for the rules is achieved through the definition of attributes for non-terminal symbols by associating them with each production. This way, they are defined to manipulate attributes, which are associated with the grammar symbols.

The lexical and syntactical phases of processing precede the semantic. The lexical analysis has as the main goal to analyse the inputs and convert them into a set of terminal symbols. The result of this processing is used in the syntactical phase to generate an $\mathrm{Ab}$ stract Syntax Tree (AST) (Bürger et al., 2010). These two first phases can be applied to intelligent environments once this kind of domain should be filled with sensors sending raw data to be analysed by a contextaware system. Then, the semantic analysis is performed with the goal of taking unevaluated attributes as input and returning evaluated ones.

Another characteristic of AGs is the possibility of manipulation of the attribute values at any node of the tree (Bürger et al., 2010). This feature is very important once in an intelligent environment the states of entities (attribute values in AGs) may change frequently and their values must be available to be used by the system at any time, with different purposes. A generic Abstract Syntax Tree of an intelligent environment is presented in figure 1 .

Te terminal symbols, in a, represent the raw data sent by sensors from the intelligent environment, in b. It refers to any sort of data captured by the sensors (e.g. humidity, level of lightness, localization, the presence of entities in a room). It will be used to characterize new situations (new context). In the general structure a, it will be characterized as been synthesized or inherited attributes and can be analyzed in different nodes of the tree. The entities represent the non-terminal symbols in an AG and may assume all the characteristics of them. They can be composed of several data properties (attributes). Besides that, attribute values from one entity may be used by other entities. In these cases, they will assume synthesized or inherited features. From the generic AST, it is possible to define rules to validate the structure that will be applied context-aware system.

Thus, it is possible to state that AGs can contribute to the consolidation of intelligent environments through different approaches. In this paper, it will be addressed the possibility of applying AGs for the validation of activities of users.

\section{Grammar for human activity recognition}

This section has as the main purpose to present an attribute grammar for validation the structure of the users' activities recognized in an intelligent environment. The grammar has the following structure:

$$
\begin{aligned}
& \text { p1: activity } \rightarrow \text { tasks, activityDescription } \\
& \text { p2: tasks } \rightarrow \text { task } * \\
& \text { p3: } \text { task } \rightarrow \text { subtasks, taskDescription } \\
& \text { p4: subtasks } \rightarrow \text { subtask } * \\
& \text { p5: } \text { subtask } \rightarrow \text { name, location } \\
& \text { p6: } \text { taskDescription } \rightarrow \text { name, location, begin } \\
& \text { p7: activityDescription } \rightarrow \text { code, name, } \\
& \text { location, resource, begin, duration } \\
& \text { p8: duration } \rightarrow \text { TEXT, begin } \\
& \text { p9: name } \rightarrow \text { name } 1, \text { location }
\end{aligned}
$$

Considering that every activity performed by users can be seen as a sequence of actions, for this attribute grammar it was defined that an activity can be composed of a set of tasks and an activityDescription (p1, p2). These tasks refers to small actions that the user has to perform before execute the activity itself. Each of these tasks may be composed of a set of subtasks, which are smaller actions that need to be concluded in preparation for the task to be achieved and a taskDescription (p3, p4). Each subtask is composed of its name and location where it should be performed (p5). The taskDescription includes the task's name, location and hour of starting (p6). The activityDescription refers to the attributes that are considered to be relevant, namely, an identification code, the name of the activity, the location where the user intends to perform it, a set of resources that will be necessary for the user to accomplish it, the hour that he started (begin) and the duration of the activity (p7). The duration of an activity is defined by the current time (TEXT) and the starting time (p8). At last, the names of activities result from the names of each task that compose them and their location. The names of tasks result from the subtasks' names and location (p9).

Figure 2 presents an example applied to a specific activity named readBook. It shows an AST that represents how the grammar was developed and how it will be used to validate the activities.

The values of the attributes in this example refer to data acquired from sensors. After properly analysing this raw data, the system fills the structure that will be used by the grammar to validate the activities. Thus, the attributes of the symbols in the grammar are represented by context data.

These preparation steps that the user accomplish before performing an activity are naturally necessary, thus, it is important for the system to understand them. In Figure 2, it is possible to identify that the structure of the activity readBook has one set with two tasks that must be complete before (and during) the performance and one subtask that represents the preparation for its development.

Considering the structure of the grammar and the values of attributes detected by sensors for the activity of readBook, the productions will have the following behaviour:

p5: subtask $\rightarrow$ goToShel $f$, Hallway

p6: taskDescription $\rightarrow$ takeBook, Shelf $, 12: 00$

p6: taskDescription $\rightarrow$ Sit,ArmChair1, $12: 01$ 


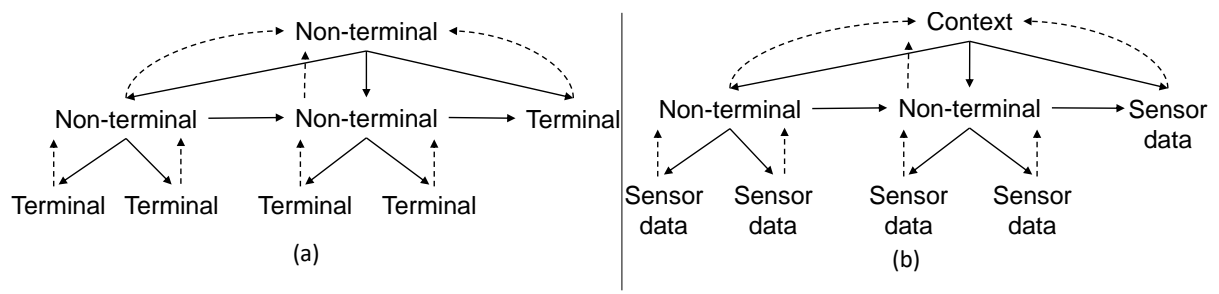

Figure 1 a General structure of AST. b Generic AST for intelligent environments.

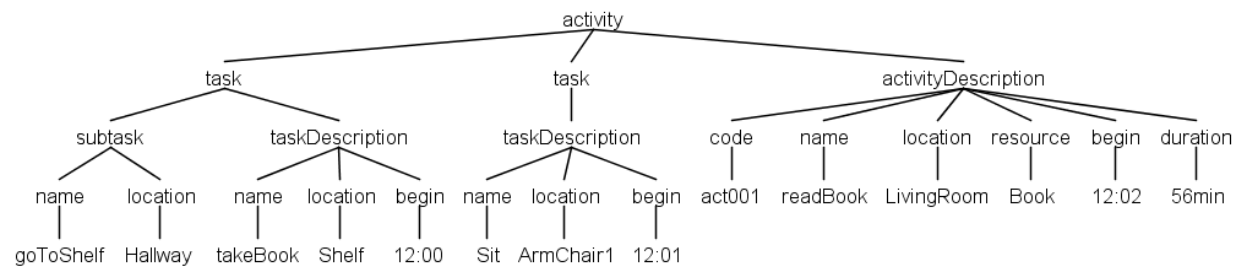

Figure 2 Abstract Syntax Tree of the human activities recognition grammar.

p7: activityDescription $\rightarrow$ act 001 , readBook, LivingRoom, Book, $12: 02,56 \mathrm{~min}$

All the productions related to terminal symbols in the grammar will have there structure filled by sensor data. As can be seen, p6 is executed twice, since the activity is composed by two tasks (takeBook and Sit).

The composition of activities in intelligent environments is performed through a definition of a set of attributes that are considered to be relevant for them to be accomplished. For this, the system should have a module with machine learning algorithms aiming identification of patterns of user's behaviour. Through the understanding of the steps that the user usually performs by accomplishing a set of tasks (activity), it is possible to identify what context data the system should consider to model new activities. For instance, by analysing the user's routine activities the system identifies that usually when he goes to the shelf in the hallway, he also takes a book, sits on the armchair and spends some time there, it is possible to infer that this flow of actions has a considerable probability of being part of an activity. From the analysis of this set of parameters, it is possible to classify it as being part of the activity of reading. The AG will use this data as synthesized and inherited attributes for the symbols for the validation of the structure of the activity.

The validation is done through the analysis of each of the steps that composed them, i.e., if their tasks and subtasks are finished within the expected parameters. Besides that, the system identifies the conclusion of activity by analysing the behaviour of the user. It considers the time used to finish the tasks, the resources, location and any other relevant information. If the values of these attributes are within a predefined acceptable range, the system can infer that the activity as concluded with success.

The mapping and monitoring of activities are important because any changes identified during the execution of an activity, task or subtask, may be related to problems with the user, for example, if considering an Ambient Assisted Living domain, the unexpected changing of behaviour can characterize health problems.

\subsection{Semantic rules}

One of the main advantages of applying attribute grammars to intelligent environment relays in the possibility of creating semantic rules that can be used to properly manipulate context data. For the development of this research it was defined that each activity is the result of a set of tasks and other additional parameters. And, each of the tasks is the result of a set of subtasks and additional parameters. The following list presents some examples of semantic rules defined for the activities recognition grammar.

1. activityDescription.duration $=$ getCurrentTime (TEXT.value) - activityDescription.begin;

2. activityDescription $\cdot$ begin $=$ getTime(TEXT.value $)$;

3. activityDescription.resource $=$ TEXT.value;

4. activityDescription.location $=$ TEXT.value;

5. activityDescription.name $=$ taskDescription1.name (taskDescription2.name)*,

taskDescription1.location(taskDescription2.location)*;

6. activityDescription .code $=$ TEXT.value;

7. taskDescription.begin $=$ getTime(TEXT.value);

8. taskDescription.location $=$ TEXT.value;

9. taskDescription.name $=$ subtask1.name (subtask2.name)*, subtask1.location(subtask2.location)*;

10. subtask.location $=$ TEXT.value;

11. subtask.name $=$ TEXT.value;

The first rule (1) refers to the calculation of the time spent by the user to finish a specific activity. This rule allows the system to identify changes in the behaviour of the user while performing that activity. Thus, with the information of this parameter, the system could infer the impact that this can have to the user. Besides the TEXT.value referring to the current time, this rule uses an inherited attribute related to the starting time of the activity (rule 2). Applying these rules to the activity of readBook, the structure is the following: 
1. activityDescription.duration = getCurrentTime (TEXT.value) - 12:02;

2. activityDescription. begin $=12: 02$;

The third rule (3) contains the data related to any resource used by the user to perform the activity. The resources used and his location are fundamental for the system to adapt its applications in order to create a better environment, aiming to assist him. In the readBook activity, this rule has the following structure:

3. activityDescription.resource $=$ Book;

The location of the user is relevant for the system to understand his intentions (rules 4, 8 and 10). The name of the activity is defined through the analysis of the tasks involved in preparation to it and where they were performed (rule 5). In the readBook activity:

4. activiyDescription.location $=$ LivingRoom;

8. taskDescription.location $=$ Shelf;

8. taskDescription.location $=$ Shelf;

8. taskDescription.location $=$ Armchair 1 ;

10. subtask.location = Hallway;

Rules number 2 and 7 allow the grammar to identify the exact time the user started an activity or task. Rules 6 and 11 are generated by the system and in the readBook activity will have the structure:

6. activityDescription $\cdot \operatorname{code}=$ act 001 ;

11. subtask.name $=$ goToShelf

The productions of the semantic rules can be used as the basis for the analysis of the level of certainty of specific contexts. This means that they can contribute to the minimization of the problem of uncertainty. For instance, the system should be aware of the time spent by the user to finish the activities. The following code describes how production could be allied with context analysis.

if (getDuration(activity.duration) not in expecDurValues [ ])

then analyse (activity.duration)

The result produced by rule 1 should be analysed according to a pre-defined range of values, referring to the time. If the production does not have a value within the expected set, this might mean that there are problems related to the activity. For instance, the value of sensorData.TIME.now was not captured with precision, resulting in an uncertain time spent or it may be related to problems with the user, once he is not been able to finish the task as usual. To solve this problem all the process of the activity.duration rule should be executed again.

\section{Related Work}

One of the main contributions of this work is the description of an attribute grammar applied to intelligent environment. At least to the best of the authors' knowledge, there are no similar approaches relating these two fields of research. Thus, aiming to describe the feasibility of using this approach, it is presented in this paper a case study of modelling of activities. There are works in the literature with relevant contributions that can be related to the one presented here, even though not using attribute grammars to model human activities. Most of them apply decomposition of tasks into subtasks and actions, creating a hierarchy and considering temporal aspects.

In (Giese et al., 2008), the authors states that formal modelling of tasks help users to perform activities with computational support. For instance, it is possible to define rankings of priority of activities to be performed according to the user's context, considering, for example, safety and social skills. The authors consider three parameters: simulation of time and conditions, referring to preconditions for tasks and possible obstacles; spatial behaviour, that analyses the location where the tasks will be performed, the objects used and the possibility of changing actors from one location to another, and; analysing communication, referring to the hierarchy and dependence.

In (Bolton et al., 2011), it is described the syntax and semantics of a function model capable of integrating task analysis with formal verification called EOFM (Enhanced Operation Function Model), based on Extensible Markup Language (XML). Changes related to activities are standardized by the model and it defines the behaviour of them according to their goals, based on the analysis of state and derived variables.

In (Mori et al., 2002), it is presented the ConcurTaskTree Environment (CTTE), which's goal is to provide means for management of task models for cooperative applications. Among the features, it is interesting to highlight: clear definition of temporal logics for task and their relations; the creation of categories such as user tasks (human routine activities), application tasks (related to the system), interaction tasks (relating user and the system) and abstract tasks (tasks belonging to more than one category) (Mori et al., 2002).

\section{Discussion and final considerations}

Different formalisms are been used to validate proposals for improvement of intelligent environments. An attribute grammar is an alternative due to the strict formalism for the definition of its symbols and attribute values. It allows the specification of semantic rules providing meaningful characteristics for the grammar. This creates a strong bond with context-aware applications. Thus, it is possible to create another layer of validation for the formal representations of real-world situations.

Another contribution of this work refers to the formalism provided by attribute grammars that can be extended to other ends, for instance, Ambient Assisted living, House Automation and Intelligent Vehicles. This can be evidenced once, in spite of the domain that an attribute grammar is being applied, its structure will have all necessary arguments, e.g., symbols, attribute values and production of semantic rules.

Context-aware systems often face the problem of uncertainty of information. This may be caused by problems with hardware of sensors preventing them to send updated 
data to be manipulated. Another source is related to design problems. This makes the system to not understand precisely what it should do with context data. Uncertainty can be faced by increasing the amount of context data that is used by the system. For instance, installing more sensors to monitor the environment and its users, or applying new algorithms of machine learning and inference rules aiming to increase the knowledge of the system (Tian et al., 2018). Besides that, the use of propositional logic to create rules is a useful approach. However, in many cases it is impossible to map all the consequences for a given premise due to the very wide range of possibilities it can have (Russell and Norvig, 2009). According to (Burgman, 2005), one way of minimizing uncertainty is through the analysis of knowledge models seeking for all possible alternatives of assumptions, regarding specific contexts and defining probability weights for each of the possible outcomes.

According to (White et al., 2018), the definition of uncertainty models help the identification of its source and the definition of optimal solutions for decision making. They can generate unique outputs based on the processing of imprecise data. This can be applied to context-aware situations formalized through an attribute grammar approach when the data from sensors are not enough to define a situation.

It is believed that attribute grammars can be allied to other fields of artificial intelligence, e.g., machine learning algorithms. Thus, the next step of the project is to merge them aiming the improvement of solutions for contextaware systems. At last, real-world tests are still needed for the validation of this approach.

\section{Acknowledgements}

"This work has been supported by FCT - Fundação para a Ciência e Tecnologia within the Project Scope: UID/CEC/00319/2019."

\section{REFERENCES}

Anderson, M. P., Woessner, W. W., and Hunt, R. J. (2015). Chapter 10 - forecasting and uncertainty analysis. In Anderson, M. P., Woessner, W. W., and Hunt, R. J., editors, Applied Groundwater Modeling, pages 443491. Academic Press, San Diego, 2nd ed. edition.

Bolton, M. L., Siminiceanu, R. I., and Bass, E. J. (2011). A systematic approach to model checking human-automation interaction using task analytic models. IEEE Transactions on Systems, Man, and Cybernetics - Part A: Systems and Humans, 41(5):961-976.

Burgman, M. (2005). Risks and Decisions for Conservation and Environmental Management. Ecology, Biodiversity and Conservation. Cambridge University Press.

Bürger, C., Karol, S., and Wende, C. (2010). Applying attribute grammars for metamodel semantics. ECOOP 2010 Workshop Proceedings - International Workshop on Formalization of Modeling Languages, FML'10.

Freitas, L., Henriques, P. R., and Novais, P. (2018). Uncertainty in context-aware systems: A case study for intelligent environments. In Álvaro Rocha, Adeli, H., Reis, L. P., and Costanzo, S., editors, Trends and Advances in Information Systems and Technologies, WorldCist2018, volume 745 of Advances in Intelligent Systems and Computing, pages 225-231. Springer International Publishing, 1 edition.

Giese, M., Mistrzyk, T., Pfau, A., Szwillus, G., and von Detten, M. (2008). Amboss: A task modeling approach for safety-critical systems. In Forbrig, P. and Paternò, F., editors, Engineering Interactive Systems, Berlin. Springer Berlin Heidelberg.

Knuth, D. E. (1968). Semantics of context-free languages. In In Mathematical Systems Theory, pages 127-145.

Mori, G., Paterno, F., and Santoro, C. (2002). Ctte: support for developing and analyzing task models for interactive system design. IEEE Transactions on Software Engineering, 28(8):797-813.

Russell, S. and Norvig, P. (2009). Artificial Intelligence: A Modern Approach. Prentice Hall Press, Upper Saddle River, NJ, USA, 3rd edition.

Tian, W., Heo, Y., de Wilde, P., Li, Z., Yan, D., Park, C. S., Feng, X., and Augenbroe, G. (2018). A review of uncertainty analysis in building energy assessment. Renewable and Sustainable Energy Reviews, 93:285 301.

White, J. T., Fienen, M. N., Barlow, P. M., and Welter, D. E. (2018). A tool for efficient, model-independent management optimization under uncertainty. Environ. Model. Softw., 100:213 - 221. 